\title{
GENERACIÓN DE VALOR AGREGADO EN LAS PYMES A TRAVÉS DE LA INNOVACIÓN EMPRESARIAL
}

\author{
Ebor Fairlie Frisancho* \\ E-mail: efairlie@unmsm.edu.pe
}

\begin{abstract}
RESUMEN
El presente estudio de Investigación ha evaluado a pequeñas y microempresas de los distritos de Villa El Salvador, San Juan de Miraflores, San Martín de Porres, San Juan de Lurigancho y La Victoria, específicamente el Conglomerado de Gamarra. La muestra fue de 20 entrevistas en cada distrito y 5 por cada tipo de producto. La muestra se tomó al azar dependiendo de los productos elegidos: artesanías, carpintería/muebles, confecciones y cuero/calzado. La entrevista a los dueños y/o encargados de las empresas se determinó en base a preguntas diseñadas y revisadas por expertos, presentando validez y confiabilidad.
\end{abstract}

Las comparaciones en general demostraron que un 82\% de PYMES que han aplicado algún tipo de innovación en su empresa en los últimos dos años han visto incrementadas sus ventas en relación a sus competidores, mientras que aquellas que no lo hicieron han tenido un incremento de ventas del $27 \%$.

Palabras clave: Valor agregado, innovación, PYMES, ventas.

\begin{abstract}
The present study has evaluated small and micro-businesses of the districts of Village El Salvador, San Juan de Miraflores, San Martin of Porres, San Juan de Lurigancho and in La Victoria (Gamarra). The sample was of 20 in each district and 5 by each type of product.

The sample it takes depending on the chosen products: crafts, carpentry/movable, making and leather/ footwear. The interview to the owners and/or responsible for the businesses was determined in base to questions designed and revised by experts, presenting validity and confiability.

The comparisons in general showed that a $82 \%$ of PYMES that have applied some type of innovation in its business in the last 2 years they have seen increased its sales relating to its competitors, while those that it did not they do, alone an increment in the sales in a $27 \%$ of them has been reflected.
\end{abstract}

Keywords: Aggregate value, innovation, PYMES, sales.

\footnotetext{
Doctorado en Administración. Magister en Administración. Licenciado en Administración de Empresas. Profesor auxiliar e investigador de la Unidad de Investigaciones de la Facultad de Ciencias Administrativas. Asesor de la Oficina de Calidad Académica y Acreditación de la Facultad de Ciencias Administrativas (UNMSM).
} 


\section{INTRODUCCIÓN}

La innovación realizada en cualquier sector trae una mejora tanto de bienestar social como económico. No es que no querramos innovar sino que tenemos la obligación de hacerlo o pereceremos en el intento. Hoy en día son numerosas las empresas que están invirtiendo recursos en Investigación y Desarrollo, pero esto requiere tiempo y sobre todo una cantidad de dinero que a la larga pudiera no rendir los frutos esperados, convirtiéndose en un "gasto" lo que realmente es una inversión. Entonces ¿que les espera a las Pequeñas y Medianas Empresas (PYME) si no cuentan con el dinero para ello?

En nuestro país las PYMES son generadoras de empleo; inclusive en las universidades se están cambiando los planes de estudio para que los estudiantes puedan egresar con planes de negocio terminados que les permitan poner en marcha sus propias empresas, convirtiéndose así en generadores de empleo y no en simples empleados. Aunque en nuestro país existen programas de generación de empresas y capacitación, el apoyo que se brinda a las PYMES todavía es limitado, pese a que este tipo de empresas son las que más utilizan los insumos locales y la mano de obra tradicional.

Hoy en día ya se está hablando de Tecnologías de Información y Comunicación (TIC) como una herramienta imprescindible para el acceso y comunicación con nuevos mercados, en especial con los internacionales, además de facilitar y mejorar la toma de decisiones en línea, optimizando las relaciones con proveedores y clientes.

\section{DISEÑO EXPERIMENTAL, MARCO TEÓRICO Y PLANTEAMIENTO DE HIPÓTESIS}

El estudio de investigación es aplicativo y no tiene conexión con programas de investigación en marcha. Fue realizado en los distritos de Villa El Salvador, San Juan de MIraflores, San Martín de Porres, San Juan de Lurigancho y La Victoria. La muestra consideró 20 entrevistas en cada distrito y 5 por cada tipo de producto.

La innovación es un factor que determina el avance de una empresa y por lo tanto incrementa las capacidades de la misma. Innovar no es colocar un nuevo producto, comprar una nueva maquinaria o contratar una persona o empresa que descubra las novedades existentes hoy en día; en verdad es mucho más complicado que eso. La innovación empresarial fortalece los procesos gerenciales clave de la organización y le da un valor agregado propio, que es lo que diferencia a una empresa de sus competidores.

Es sabido que las PYMES no cuentan con el dinero suficiente para subsidiar los cuantiosos gastos de investigación que representan este tipo de estudios y ahí es donde el Estado interviene en forma contundente con el apoyo Centro de Promoción de la Pequeña y Micro Empresa (PROMPYME) para la capacitación, estudio y promoción de las pequeñas y microempresas de nuestro país, cuyos resultados son difundidos con el apoyo de instituciones públicas y privadas.

Las universidades no se quedan atrás. Las investigaciones y publicaciones generadas por el personal docente sirven de base para su aplicación en el mercado. Los productos elegidos son estratégicos para el desarrollo económico de las PYMES de los distritos y emporios indicados, asi como los de mayor venta y solicitud por parte de los clientes. Inclusive, en algunos casos, grandes empresas transnacionales compran y/o mandan a desarrollar productos a estas PYMEs en diversos colores, diseños y tamaños, demostrando que existen una gran gama de clientes que tratan de tener productos diferenciados $\mathrm{y} / \mathrm{o}$ con innovaciones.

Las hipótesis planteadas fueron:

H1: La generación de valor agregado incentiva a las PYMES a un mayor desarrollo sostenido.

H2: Hay diferencias significativas entre las PYMES que han incorporado algún tipo de innovación empresarial desde su creación y las que no lo han hecho.

H3: La innovación tecnológica es una de las más utilizadas en Lima.

Los objetivos planteados para la presente investigación son:

- Precisar el mayor o menor valor agregado de la PYMES en la ciudad de Lima para generar conocimiento que aporte, en forma significativa, propuestas para su desarrollo.

- Determinar si existe una relación estadística significativa entre la innovación em- 
presarial y la generación de valor agregado.

Las entrevistas realizadas incluían 15 preguntas y se efectuaban personalmente a los dueños y/o encargados de cada empresa entrevistada. Se estima que el tiempo necesario para completarla debe ser de 15 a 20 minutos.

\section{MÉTODOS Y RESULTADOS}

El universo total de empresas formales al 2004, según la Superintendencia Nacional de Administración Tributaria (SUNAT), es de 659,046 organizaciones, de las cuales se desprende el siguiente cuadro:

Cuadro № 1. Perú Urbano: Distribución de empresas formales según tamaño (2004).

\begin{tabular}{|l|r|r|}
\hline \multicolumn{1}{|c|}{ Tipo de empresa } & Número de empresas & \multicolumn{1}{c|}{$\%$} \\
\hline Micro empresa 1/ & 622,209 & $94.4 \%$ \\
Pequeña Empresa 2/ & 25,938 & $3.9 \%$ \\
Gran Empresa y mediana 3/ & 10,899 & $1.7 \%$ \\
Total & $\mathbf{6 5 9 , 0 4 6}$ & $\mathbf{1 0 0 . 0 \%}$ \\
\hline
\end{tabular}

Fuente: SUNAT. Declaración de Renta 2005 III Censo Nacional.

$1 /$ Ventas anuales menores a US $\$ 80,000$

$2 /$ Ventas anuales entre US $\$ 80,000$ y US $\$ 750,000$

$3 /$ Ventas anuales mayores a US $\$ 750,000$. El total de las medianas es un valor

estimado. No es posible desagregar esta categoría según su nivel de ventas.

Elaboración: PROMPYME.

De acuerdo a PROMPYME, sólo en Lima y Callao existen 314,339 PYMES ubicadas en los diferentes distritos. De esta cantidad, en los distritos de Villa El Salvador, San Juan de MIraflores, San Martín de Porres, San Juan de Lurigancho y La Victoria (Gamarra) se encuentran aproximadamente 75,000 PYMES, es decir un 19\% del total de empresas.

Gráfico $\mathbf{N}^{\circ}$ 1. Porcentajes de distritos entrevistados.

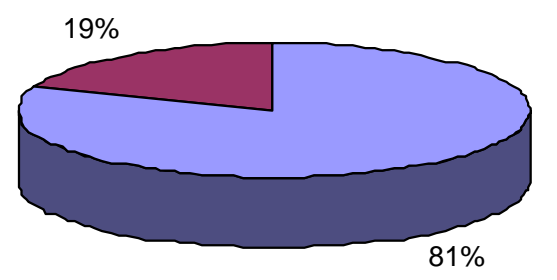

$\square$ Lima $\square$ distritos entrevistados

Para la confiabilidad de la prueba se ha utilizado el análisis de ítems y el método de consistencia interna a través del coeficiente Alpha de Cronbach. Dicho análisis alcanzó un valor de 0.67 porque lo que se puede concluir que la prueba permite obtener puntajes relativamente confiables.
Para recoger la muestra se estableció la elección al azar de empresas de los rubros mencionados anteriormente.

Los permisos necesarios se solicitaron oportunamente a la Dirección Académica de la Facultad para poder tener acceso a las empresas elegidas, previa coordinación con los dueños de mismas y/o que mostraran predisposición a ser entrevistados.

El tamaño de la muestra de investigación es de 20 empresas por distrito o emporio, es decir 100 PYMES, por lo que el resultado genera un nivel de confianza de $99 \%$ con respecto a los resultados (la muestra mínima es $2 \%$ ), con un margen de error de $0.05 \%$.

\section{ANÁLISIS Y DISCUSIÓN}

\section{ANÁLISIS}

De la entrevista, se han rescatado las preguntas cuyos resultados han sido los más significativos, los que a continuación se detallan:

- Por diversos motivos, un 95\% de las empresas entrevistadas se encuentra en la continua expectativa de generar valor agregado al cliente, de las cuales un $70 \%$ toman en cuenta las sugerencias de los clientes y un $30 \%$ se han informado sobre agregados adicionales en los productos. 
- Con respecto a la antiguiedad de la empresa, un $30 \%$ tiene entre uno y cinco años, y un $35 \%$ tiene entre cinco y diez años. Solo un $20 \%$ de las empresas tienen más de diez años de funcionamiento.

- De la entrevista se estima que un $65 \%$ de los trabajadores de sexo masculino contribuyen en generar mayor valor agregado a los productos, a comparación de un $45 \%$ de las trabajadoras.

- De las empresas que han incorporado algún tipo de innovación en su empresa, un $45 \%$ ha realizado una innovación tecnológica, un $38 \%$ innovación del producto y un $18 \%$ innovación administrativa.

- El $40 \%$ de las empresas entrevistadas indican que están innovándose continuamente, independientemente del rubro al que pertenecen. Un 31\% demora entre uno y dos años para innovar sus productos y un $25 \%$ lo hace cada tres años o más.

- De los que se innovan constantemente, el $45 \%$ de ellos realiza algún tipo de innovación tecnológica.

- Un $15 \%$ de los entrevistados afirma haber obtenido algún tipo de apoyo del Estado para su PYME, a comparación de un $85 \%$ que no lo ha tenido.

\section{DISCUSIÓN}

Los resultados con las entrevistas indican que éstas cumplen con los requisitos básicos, pues la confiabilidad por consistencia interna alcanza un coeficiente de Kuder Richardson 20 de 0.62 (Alpha de Cronbach).

Podemos observar que la mayoría de las empresas presenta un valor agregado constante, y continuamente están buscando dar solución a las expectativas del consumidor.

Los hombres, en su mayoría, generan un alto valor agregado a los productos ya que aportan más sugerencias. Hay que recordar que en los rubros elegidos (artesanías, carpintería/muebles y cuero/calzado) trabajan un $75 \%$ de hombres. En confecciones existe un 93\% de mujeres dedicadas a este rubro.

Un elemento muy interesante es que de los entrevistados, un $89 \%$ se compone de empresarios hombres y solamente un $11 \%$ de mujeres.
Se muestra adicionalmente que la orientación a la innovación tecnológica es constante, y el entrevistado considera que es un elemento preponderante en su desarrollo para hacer frente a la fuerte competencia, además de las facilidades que se están dando a las PYMES para que accedan a la innovación.

\section{CONCLUSIONES}

1. Las Pequeñas y Micro Empresas (MYPES) están en constante innovación debido a la alta competencia de los productos que elaboran.

2. La mayoría de empresarios son de sexo masculino a pesar de las facilidades que existen para las mujeres empresarias, en especial en algunos rubros como confecciones.

3. La mayoría de las innovaciones planteadas se realizan por sugerencias de los clientes en especial del público en general, y una minoría por los mismos trabajadores o dueños que son informados de nuevos adelantos tecnológicos utilizados en el rubro.

4. La innovación tecnológica es la más utilizada, en especial para el desarrollo de sus productos, seguida de la parte administrativa con el uso de sistemas de información.

5. Las diferencias en ventas entre empresas del mismo rubro con respecto a aquellas que se innovan constantemente, es significativa.

\section{BIBLIOGRAFÍA}

Autoridad Autónoma del Proyecto Especial Parque Industrial del Cono Sur, Villa El Salvador (1999). Agencia Española de Cooperación Internacional. Parques Industriales de Desarrollo: una propuesta práctica y la experiencia de un parque industrial: $\mathrm{El}$ caso del Parque Industrial del Cono Sur. Villa El Salvador. Lima.

Aznarán, G. (2003). "Propuestas de generación de empleo". Gestión en el Tercer Milenio, 12, pp. 113-117.

COFIDE (1998). Directorio empresarial del Parque Industrial Villa El Salvador. Lima.

El Comercio - Universidad San Martín de Porres (2000). Innovación (fascículo) Tu propio negocio. Lima. 
Congreso de la República (2003). La pequeña y microempresa en el desarrollo nacional. Lima.

COPEME (2004). La microempresa en el Parque Industrial de Villa El Salvador. Compendios Estadísticos. Lima.

Dirección de Desarrollo Económico de la Municipalidad de Villa El Salvador (2004). La microempresa en el Parque Industrial de Villa El Salvador, datos estadísticos. Lima.

Espinoza, Nemesio (1999). "Estrategias de desarrollo de las PYMES. La formación de empresarios en las universidades". Revista
Gestión en el Tercer Milenio, año 1, № 1, pp. 101-110.

Espinoza, Nemesio (2001). "Las innovaciones tecnológicas en las PYMES”. Revista Gestión en el Tercer Milenio, año 4, № 7, pp. 24-33.

Hernández, R.; Fernández, C. y Baptista, P. (2001). Metodología de la investigación. México, MxGraw-Hill.

Kenneth, Hopkins y Glass (1997). Estadística básica para las ciencias sociales y del comportamiento. México, Prentice-Hall Hispanoamérica. 